\title{
Plataforma de Evaluación de Mecanismos de Localización de Nodos en Redes de Sensores Inalámbricos Bajo el Agua - UWSN
}

\author{
Platform for the evaluation of node location mechanisms in wireless \\ underwater sensor networks - uwsn
}

Jorge Luis Paucar Samaniego ${ }^{1}$, Osvaldo Geovanny Martínez Guashima. ${ }^{2}$, Fausto Francisco Navarrete Chávez ${ }^{3} \&$ Gisela Cujilema Daga. ${ }^{4}$

\section{Recibido: 30-05-2019 / Revisado: 28-06-2019 /Aceptado: 30-07-2019/ Publicado: 06-09-2019}

\begin{abstract}
.
DOI: https://doi.org/10.33262/cienciadigital.v3i3.3.777

In this researching, it is possible to do the evaluation of mechanisms of localization of nodes in Networks of Wireless Sensors Under Water (UWSN). The AquaSim simulation tool has allowed the measurement of specific variables such as: Point-toPoint Delay, Consumed Energy and Throughput same that are analyzed in a real environment such as the Galapagos Islands have allowed to determine that the protocol that relates higher performance against lower energy consumption is VBF obtaining a delay of $154 \mathrm{~W} / \mathrm{s}$.
\end{abstract}

Keywords: Electronic, sensors, telecommunication, wireless sensor networks (WSN)

\footnotetext{
${ }^{1}$ Escuela Superior Politécnica de Chimborazo, carrera de Electrónica y Automatización, Facultad de Informática y Electrónica , Riobamba, Ecuador, jlpaucar@espoch.edu.ec

2 Escuela Superior Politécnica de Chimborazo, carrera de Telecomunicaciones, Facultad de Informática y Electrónica , Riobamba, Ecuador, geovanny.martinez@espoch.edu.ec

${ }^{3}$ Escuela Superior Politécnica de Chimborazo, carrera de Física, Facultad de Ciencias. Riobamba, Ecuador. fausto.navarrete@espoch.edu.ec

${ }^{4}$ Escuela Superior Politécnica de Chimborazo, carrera de Telecomunicaciones, Facultad de Informática y Electrónica. Riobamba, Ecuador, giselacd25@gmail.com
} 


\section{Resumen.}

En esta investigación, es posible hacer la evaluación de mecanismos de localización de nodos en Redes de sensores inalámbricos bajo el agua (UWSN). La herramienta de simulación AquaSim ha permitido la medición de variables específicas como: retraso de punto a punto, energía consumida y rendimiento mismo que se analizan en un entorno real, como las Islas Galápagos, han permitido determinar que el protocolo que relaciona un mayor rendimiento contra menor consumo de energía es VBF que obtiene un retraso de $154 \mathrm{~W} / \mathrm{s}$

Palabras claves: electrónica, sensores, telecomunicaciones, red de sensores inalámbricos (WSN)

\section{Introducción.}

El 75\% de la superficie terrestre está cubierta de agua en forma de ríos, mares y océanos, grandes esfuerzos investigativos se han realizado con el fin de explorar, explotar y proteger el ambiente acuático. Debido a la importancia de sus aplicaciones y su forma particular de transmitir datos, las redes de sensores inalámbricos submarinos UWSN (Underwater Wireless Sensor Networks) han despertado un creciente interés, sin embargo, las características específicas de este tipo de redes tales como: movilidad de nodos, restricción de energía, retardo, ancho de banda limitado e interferencia, plantean grandes retos de localización y enrutamiento que necesitan de una precisión exacta y una escalabilidad funcional, para tener una red totalmente accesible.

Sus múltiples aplicaciones crean la necesidad de analizar los métodos de enrutamiento para redes inalámbricas de sensores submarinos (UWSN) con el objetivo de identificar aquel que presente las mejores características de rendimiento y bajo consumo de energía. Esta tarea se ha llevado a cabo a través de simulaciones computacionales que, ejecutadas sobre diferentes escenarios de red, permiten la evaluación estadística de sus resultados.

a) Red de Sensores Inalámbricos Submarinos (UWSN)

La red de sensores inalámbricos submarinos o bajo el agua consiste en un número variable de nodos sensores que se despliegan tanto en el agua como en la superficie para llevar a cabo tareas colaborativas sobre un entorno acuático para lo cual los nodos deben intercambiar y compartir información entre sí y con las estaciones base [1].

El agua es un medio no transparente para la luz visible por lo que la propagación de ondas por radiofrecuencia se atenúa rápidamente, las ondas ópticas ofrecen una transmisión de datos de alta velocidad, sin embargo el efecto de absorción y dispersión influyen en la señal y su precisión, finalmente por su característica de baja absorción, la comunicación bajo el 
ISSN: 2602-8085

agua utiliza señales acústicas para la transmisión de información ya que ésta, a pesar de su lentitud, ofrece un rango de transmisión más largo a menores pérdidas y con varias limitaciones que incluyen el ancho de banda que es severamente limitado, los retrasos de propagación que en magnitud representan cinco veces mayor a un canal de radio frecuencia terrestre, mayores tasas de error de bits, pérdidas temporales de conectividad y potencia energética limitada.

b) Arquitectura.

Las arquitecturas más utilizadas para redes UWSN son:

1. UWSN Bidimensional: Destinadas al monitoreo del fondo del mar, estas redes están constituidas de varios sensores colocados a grandes profundidades del océano con la ayuda de anclas.

2. UWSN Tridimensional: Se compone de una red de sensores anclados a diferentes profundidades para cubrir un área de tres dimensiones. Se destinan al monitoreo de la columna oceánica y fenómenos marinos como procesos bioquímicos, corrientes de agua, contaminación y otras aplicaciones de vigilancia.

3. Red con Vehículos Autónomos Bajo el Agua (AUVs): Los vehículos autónomos son boyas relativamente costosas y están equipadas con múltiples sensores submarinos que pueden funcionar sin ataduras o control remoto es por ello por lo que son utilizadas en aplicaciones de vigilancia del medio ambiente, estudio de recursos oceánicos entre otras. [2]

c) Aplicaciones.

Las Redes UWSN habilitan un amplio rango de aplicaciones como: monitoreo del ambiente subacuático sus características, propiedades o cualquier objeto de interés, exploración del fondo marino, prevención de desastres naturales producidos en el agua, misiones militares con el fin de encontrar minas submarinas, monitoreo y vigilancia para asegurar puertos, asistencia para navegación, etc.

d) Protocolos de Enrutamiento para UWSN.

Los protocolos de enrutamiento tienen la responsabilidad de descubrir y mantener rutas confiables durante la transmisión de datos tomando en cuenta la distancia, requerimiento de energía y tiempo de vida de la red. Adicionalmente deben ser capaces de manejar la movilidad de los nodos de una manera eficiente. El enrutamiento geográfico es un tipo de enrutamiento que se basa en información de localización geográfica donde la fuente envía sus paquetes a la ubicación del destino en lugar de la dirección de red de destino. Existen algunos protocolos de enrutamiento geográfico de entre los cuales, para el presente estudio, se ha seleccionado los siguientes: VBF, HH-VBF, FBR, SBR-DLP 
ISSN: 2602-8085

\section{Reenvío Basado en Vectores (VBF)}

En este protocolo cada paquete lleva las posiciones del transmisor, del destino y del retransmisor. La ruta para el reenvío se especifica mediante el vector de enrutamiento desde el transmisor hasta el destino. Al recibir un paquete, un nodo calcula su posición relativa al retransmisor y mide su distancia a éste y el ángulo de arribo (AOA) de la señal. De la misma manera, todos los nodos que reciben el paquete calculan sus posiciones. Si un nodo determina que se encuentra lo suficientemente cerca al vector de enrutamiento, ubica en el paquete su propia posición calculada y posteriormente lo reenvía; caso contrario, simplemente descarta el paquete. De esta manera, todos los retransmisores dentro de la red forman una tubería de encaminamiento: los nodos sensores en esta tubería son aptos para reenviar paquetes y aquellos que no están próximos a la tubería de encaminamiento no reenviaran los paquetes [3].

\section{Reenvío Basado en Vectores Salto a Salto (HH-VBF)}

El protocolo de enrutamiento basado en vectores (VBF) presenta dos grandes inconvenientes, en primer lugar, un único vector fuente-destino, una sola tubería virtual podría significar la eficiencia en el enrutamiento dentro de redes densas, de la misma manera en redes dispersas las tuberías virtuales no podrían detectar a ningún nodo para reenviar el paquete, lo cual hace una red desconectada y afecta directamente en el promedio de paquetes entregados. El otro problema es el radio de la tubería virtual de enrutamiento, éste afecta el rendimiento dentro de las redes y la efectividad en el enrutamiento lo cual lo constituye en un gran problema al momento de desarrollarlo en ambientes reales.

Para solucionar estos problemas se ha desarrollado el protocolo vector basado en reenvió salto a salto. Este protocolo utiliza los principales fundamentos del protocolo VBF, con la mejora que HH-VBF, utiliza diferentes tuberías virtuales para cada nodo que entra en el reenvió del paquete. Cada nodo puede calcular adaptarse al envió del paquete para recalcular la ruta basándose en su propia posición.

En HH-VBF, se redefine la tubería de enrutamiento virtual en una nueva creación de tuberías virtuales por saltos, en lugar de una única tubería desde la fuente hasta el destino. Este nuevo concepto de salto a salto permite expandir la probabilidad de encontrar una vía de enrutamiento en comparación con $\mathrm{VBF}$. Al recibir el paquete, el nodo calcula el vector desde el transmisor hasta el destino. De esta manera la tubería de reenvío cambia en cada salto en la red, adquiere el nombre de vector basado en reenvío salto a salto (HH-VBF) [4].

Protocolo de Enrutamiento de Haz Enfocado (FBR) 
ISSN: 2602-8085

En las redes de sensores inalámbricos submarinos el poder de trasmisión depende de la distancia que se desea cubrir y esto en muchos de los casos requiere de una fuente de poder grande pero debido al diseño de la red el consumo energético es restringido lo que lo convierte en un problema. Un primer intento para solucionarlo es limitar el rango de transmisión y, para que un paquete llegue a su destino, es necesario varios nodos retransmisores. Una segunda solución es aumentar el ancho de banda con lo cual se reduce la duración del bit y por consiguiente la energía requerida. Pero el inconveniente en estas soluciones es el limitado ancho de banda y el alto costo en la colocación de más nodos en la red lo cual impide que se pueda aplicar dichas soluciones en entornos reales. Para solucionar estos inconvenientes se ha desarrollado una metodología del enrutamiento con un sistema de control. Esta técnica de enrutamiento asume que cada nodo conoce su propia localización y la localización del nodo destino, pero desconoce la posición del resto de nodos. Aunque esta afirmación no es totalmente cierta debido a que todos los nodos dentro de la red submarina se desplazan y cambian su posición constantemente, para enfrentar esta situación se desarrolló el protocolo de enrutamiento de haz enfocado, el cual desarrolló una técnica para localizar a los nodos del próximo salto con el menor consumo energético y sin sobrecargar a la red con mensajes de control.

La transacción inicial es ejecutada al nivel de potencia más bajo y la potencia se incrementa sólo si es necesario. El control de potencia es realizado como parte integral del enrutamiento y del control de acceso medio.

Para cada nivel de potencia $P_{n}$ existe un radio de transmisión $d_{n}$. Solamente los nodos que se encuentran dentro de este radio reciben la señal a un nivel suficiente para su detección [4].

\section{Enrutamiento Sectorial con Predicción de Localización de Destino (SBR-DLP)}

SBR-DLP es un algoritmo de enrutamiento basado en la localización mediante el cual un nodo sensor no transporta ninguna información acerca de los nodos vecinos ni de la topología de red. Sin embargo, se asume que cada nodo conoce su propia posición, y los movimientos planificados del nodo destino. Hay mucho interés en las aplicaciones como la exploración y el monitoreo marino que puede requerir que el nodo destino se mueva a lo largo de la red móvil para cubrir el área entera de exploración o monitoreo. Para tales aplicaciones, los nodos destino actúan como un nodo móvil, y su movimiento es usualmente predefinido antes del lanzamiento de la red. Esto en contraste a algunas aplicaciones, donde el nodo destino puede ser fijado en la superficie del agua para actuar como una entrada o un nodo central, y es a su vez conectado a una red troncal de alta velocidad.

e) Herramientas de Simulación UWSN

Los simuladores son una potente herramienta utilizada para facilitar la investigación, su facilidad de manejo, bajo costo y las múltiples pruebas satisfactorias de redes a gran 
ISSN: 2602-8085

www.cienciadigital.org

Vol. 3, N³.3, p. 162 - 176, septiembre, 2019

escala, permiten determinar si un proyecto es o no viable, Se presenta una descripción general de los simuladores disponibles para UWSN [1]:

1. Aqua-Sim

Simula la atenuación de las señales acústicas y la colisión de los paquetes dentro de las redes de sensores subacuáticos. Soporta paquetes de simulación inalámbrica CMU. Aqua-Sim es independiente del paquete inalámbrico por lo que no es afectado por las alteraciones realizadas en el mismo. Consta de 3 módulos principales: entradas, interfaces y funciones.

2. Shawn

Es un simulador para redes de sensores personalizable de código abierto, diseñado para soportar redes a gran escala. Puede ser escrito en Java, tiene características de perseverancia y desacople de las instancias de la simulación. Estas características hacen que sea más fácil la implementación. Ha sido habilitado para trabajar con varios modelos de interfaz que pueden ser usados para controlar la simulación sin conocer con precisión la implementación necesaria para utilizarla en el mundo real. Diseñado para protocolos de alto nivel que emplean simples modelos de comunicación y permiten a dos o más nodos intercambiar mensajes.

3. UWSim

Se enfoca en simulaciones de grandes extensiones de sensores y redes adhoc, pero no consideran ningún factor que afecte a la comunicación subacuática. Está enfocado para manejar ciertos parámetros propios de las UWSN como es el restringido ancho de banda, frecuencias bajas, trasmisiones altas y memoria limitada. Se encuentra enfocado como un simulador en base de protocolo/capa.

4. VisualSense

Diseñado para representar requerimientos específicos de las redes inalámbricas, así como examinar los canales de comunicación. Está diseñado para mantener una base de componentes estructurados como modelos. Ofrece un exacto y extensible modelo de radio comunicación. El modelo de radio utilizado viene acompañado de un modelo de programación de energía general que puede ser reprogramado por los fenómenos físicos.

\section{Metodología.}

El presente trabajo de investigación se engloba dentro de una investigación experimental y correlacional puesto que se utiliza la información y conocimientos adquiridos durante las investigaciones previas, para evaluar los protocolos de enrutamiento de redes de sensores inalámbricos submarinos y determinar aquel que presente mayor rendimiento y menor 
consumo energético. El primer paso para realizar la presente investigación fue la selección de la herramienta de simulación, para lograrlo se ha considerado varias características importantes que debe poseer esta herramienta como es el tipo de licencia, facilidad de configuración o creación de nuevos protocolos, disponibilidad de información en línea, facilidad en el manejo del software, posibilidad de la generación de archivos PostScript, fiabilidad en los resultados, capacidad para simular redes extensas, cantidad de recursos necesarios para trabajar, posibilidad de soportar movilidad de los nodos y capacidad de manejar redes en 3D. Estas características han sido cuantificadas a través de la Escala de Likert donde se determinó que Aqua-Sim es la herramienta idónea para simular los protocolos UWSN.

a) Esquema de la Red UWSN a simular.

En la figura 1, se representa esquemáticamente la red UWSN que se ha propuesto para ser simulada a través de la herramienta Aqua-Sim. El esquema consta de un conjunto de nodos sensores ubicados a diferentes profundidades, estos nodos se comunican con un nodo central SINK.

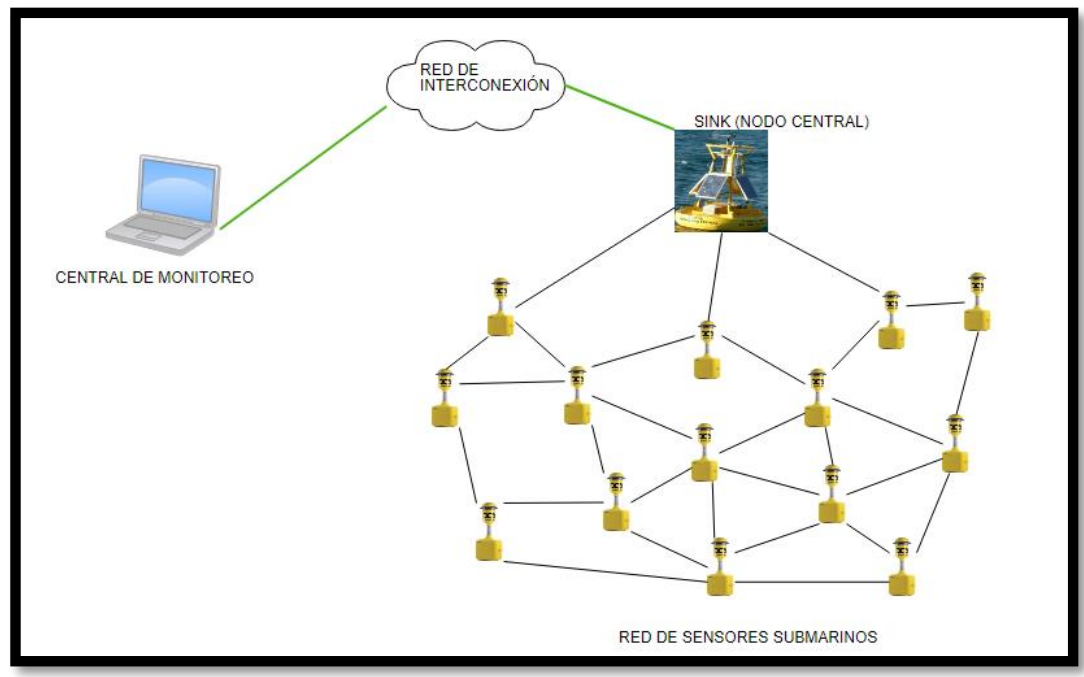

Figura 1 Modelo esquemático de la red UWSN

Fuente: Elaboración propia

b) Ambiente de Simulación.

Para el desarrollo de pruebas de funcionamiento de los protocolos y extracción de características necesarias para la configuración de la herramienta Aqua Sim, se estableció un ambiente de simulación que posee características de un entorno real.

Como modelo idóneo y con el fin de aportar para la preservación de la flora, fauna y su frágil ecosistema, se ha seleccionado a las Islas Galápagos, consideradas por la UNESCO como Patrimonio Natural de la Humanidad. Se propone la instalación de una red UWSN en el canal 
ISSN: 2602-8085

de Itabaca entre las Islas Baltra y Santa Cruz, dicho canal alberga la mayor producción de especies nutritivas de la región [6] por lo que es sumamente frágil y vulnerable a una contaminación por hidrocarburos ya que dentro de la isla Baltra se encuentra el punto de abastecimiento de combustible para toda la zona insular a través de la terminal de Aerocomercial.

c) Parámetros de Simulación.

1. Área de Cobertura: se ha establecido que el área para el desarrollo de pruebas sea de 1000 x 1000 x $500 \mathrm{~m}$ (largo, ancho, profundidad).

2. Velocidad de las corrientes: dentro de la reserva Marina Galápagos las corrientes oscilan entre $0,1 \mathrm{~m} / \mathrm{s}$ y $1,2 \mathrm{~m} / \mathrm{s}$

3. Número de Nodos: Según [3] un nodo bajo el agua brinda un alcance promedio de 100 metros en todas sus direcciones, por lo que se ha determinado un total de 100 nodos para el escenario planteado

\section{Resultados.}

De las simulaciones realizadas se han extraído veinte (20) muestras de cada escenario y posteriormente, estos datos fueron ponderados, analizados y comparados entre sí se obtienen los resultados que se presentan a continuación.

a) Análisis de los Promedios de End and End Delay

En la Tabla 1, se presentan los diferentes promedios del retardo punto a punto obtenidos para las redes densas y dispersas.

\begin{tabular}{lcccc}
\hline & \multicolumn{2}{c}{ REDES DENSAS } & \multicolumn{2}{c}{ REDES DISPERSAS } \\
\hline & $\mathbf{V}=\mathbf{0 . 1} \mathbf{~ m} / \mathbf{s}$ & $\mathbf{V}=\mathbf{1 . 2} \mathbf{~ m} / \mathbf{s}$ & $\mathbf{V = 0 . 1} \mathbf{~ m} / \mathbf{s}$ & $\mathbf{V = 1 . 2} \mathbf{~ m} / \mathbf{s}$ \\
VBF & 154,48 & 149,67 & 90,98 & 81,98 \\
HH-VBF & 221,94 & 208,26 & 147,31 & 51,23 \\
SBR-DLP & 720,78 & 561,73 & 130,02 & 137,91 \\
FBR & 169,94 & 84,385 & 4,60 & 1,49 \\
\hline
\end{tabular}

Tabla 1 Promedios End and End Delay

Fuente: Elaboración propia 
ISSN: 2602-8085

En la figura 2, se puede apreciar que, al aumentar la velocidad de los nodos en las Redes Densas, el retardo disminuye

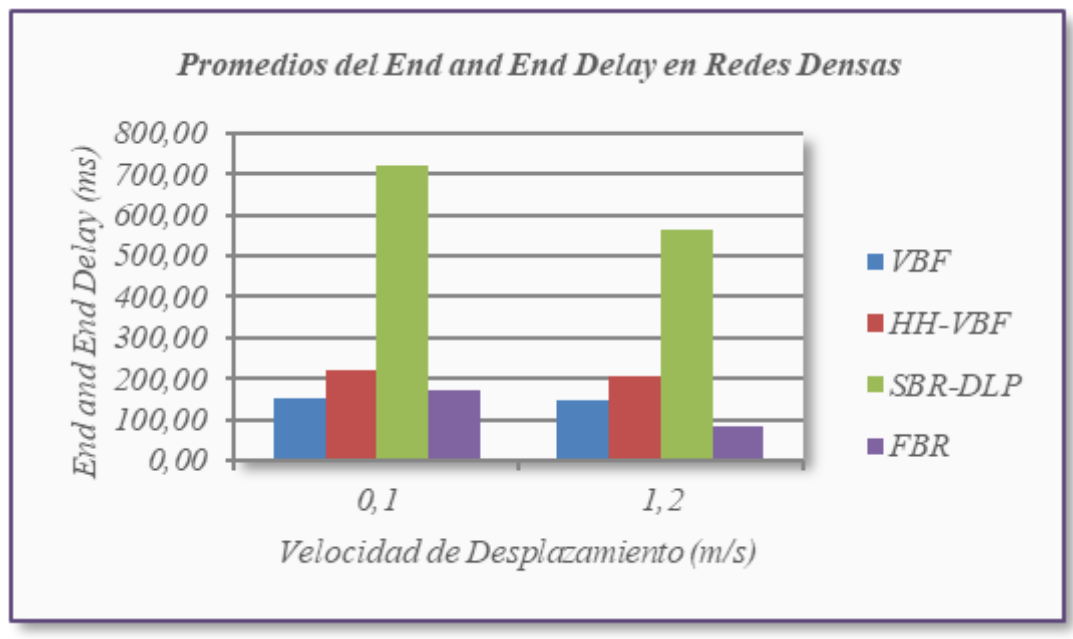

Figura 2 Promedios End and End Delay en Redes Densas

Fuente: Elaboración propia

La figura 3, se puede apreciar que, al aumentar la velocidad de los nodos en las Redes Dispersas, el retardo disminuye con los protocolos VBF, HH-VBF y FBR mientras que aumenta con SBR-DLP.

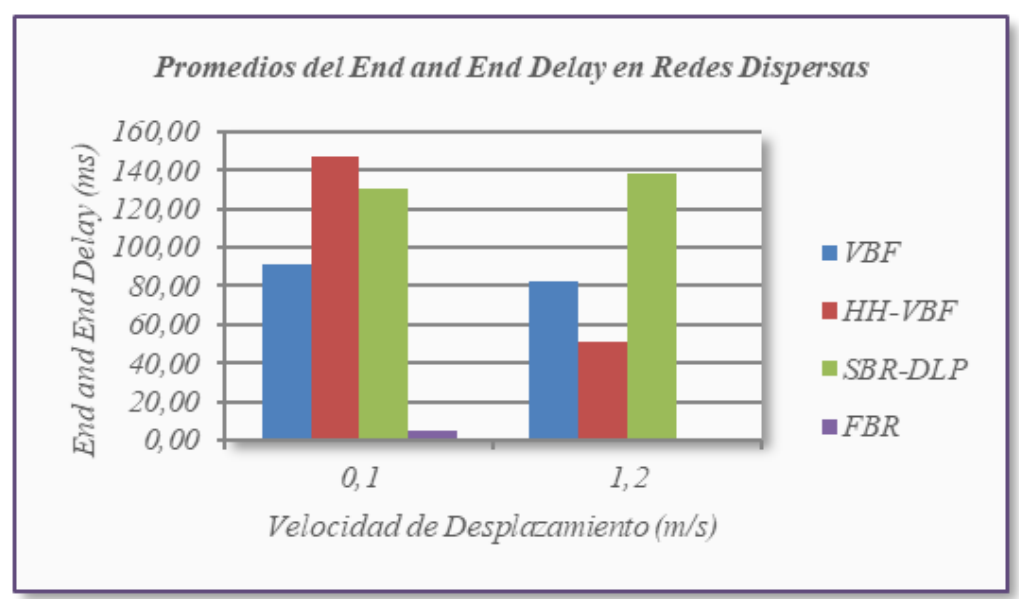

Figura 3 Promedios End and End Delay en Redes Dispersas

Fuente: Elaboración propia 
b) Análisis de los Promedios de Energy Consumption

En la tabla 2, se presenta los diferentes promedios de Energía Consumida obtenidos del escenario planteado.

\begin{tabular}{lllll}
\hline & \multicolumn{2}{c}{ REDES DENSAS } & \multicolumn{2}{c}{ REDES DISPERSAS } \\
\hline & $\mathbf{V = 0 . 1} \mathbf{~ m} / \mathbf{s}$ & $\mathbf{V}=\mathbf{1 . 2} \mathbf{~ m} / \mathbf{s}$ & $\mathbf{V = 0 . 1} \mathbf{~ m} / \mathbf{s}$ & $\mathbf{V = 1 . 2} \mathbf{~ m} / \mathbf{s}$ \\
VBF & 1,15402 & 1,15701 & 0,12683 & 0,12414 \\
HH-VBF & 2,70903 & 2,77263 & 0,31228 & 0,24371 \\
SBR-DLP & 6,69430 & 6,73551 & 0,58253 & 0,54605 \\
FBR & 1,21551 & 1,20729 & 0,12148 & 0,12156 \\
\hline
\end{tabular}

Tabla 2 Promedios Energy Consumption (W/s)

Fuente: Elaboración propia

En la figura 4, se puede observar que, para Redes Densas, al aumentar la velocidad de los nodos, el consumo energético no se ve mayormente afectado

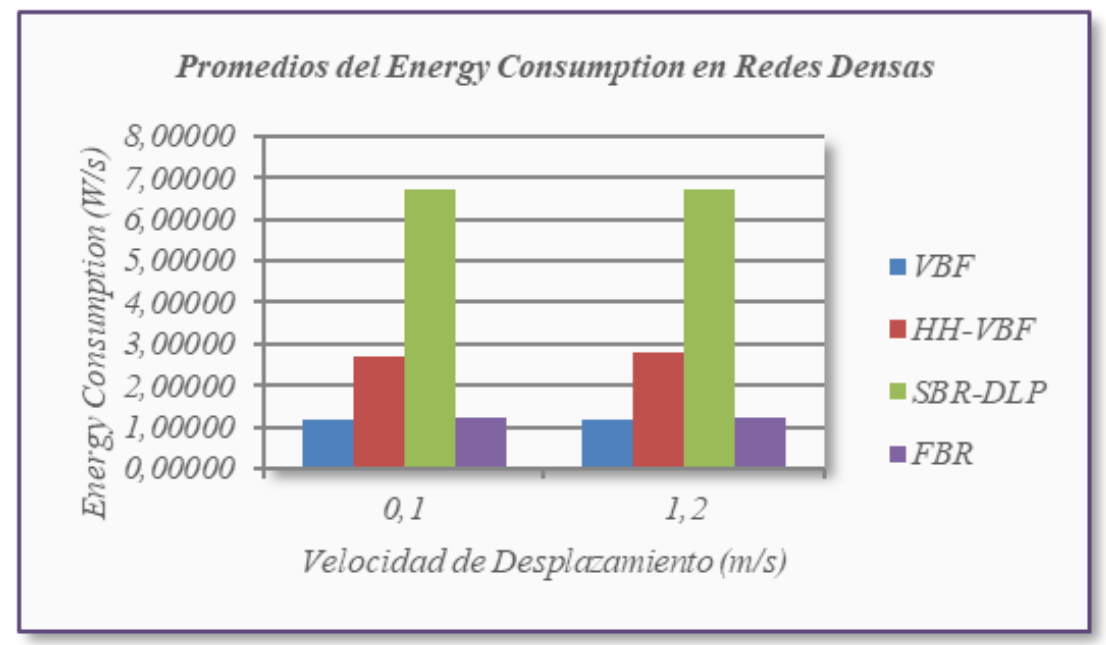

Figura 4 Promedios Energy Consumption en Redes Densas.

Fuente: Elaboración propia

En la figura 5, se puede observar que dentro de las redes dispersas al aumentar la velocidad de los nodos el consumo energético no se ve afectado significativamente con los protocolos VBF y FBR mientras que tiende a disminuir con los protocolos HH-VBF y SBR-DLP. 


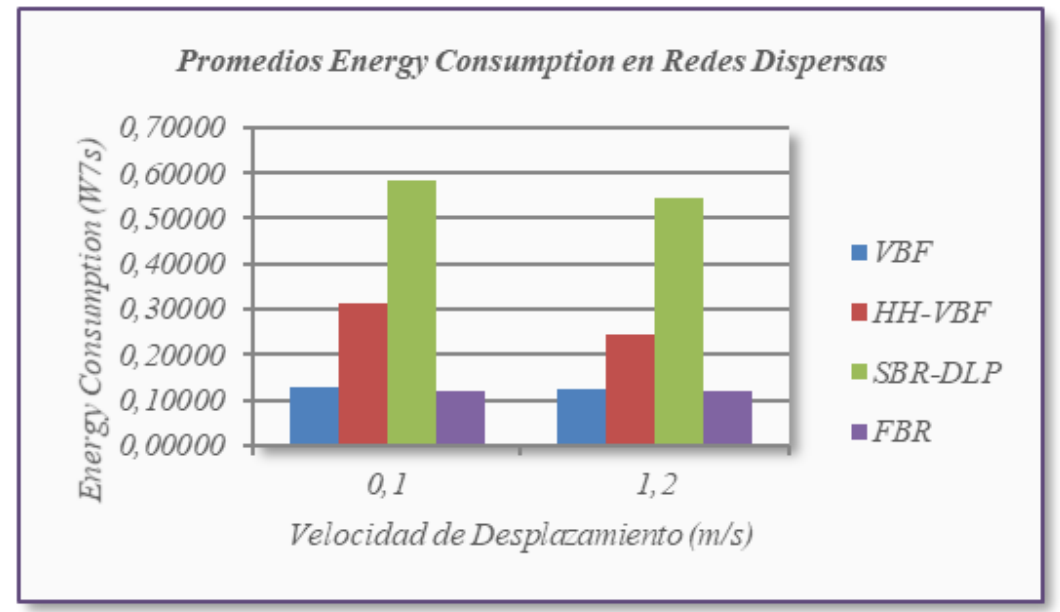

Figura 5 Promedios Energy Consumption en Redes Dispersas

Fuente: Elaboración propia

c) Análisis de los Promedios de Throughput

En la Tabla 3, se presenta los diferentes promedios del Throughput obtenidos del escenario planteado.

En el Grafico 5, se puede observar que, en las Redes Densas, al aumentar la velocidad de los nodos, el Throughput tiende a mantenerse estable en todos los casos.

\begin{tabular}{lllll}
\hline & \multicolumn{2}{l}{ REDES DENSAS } & \multicolumn{2}{l}{ REDES DISPERSAS } \\
\hline & $\mathbf{V = 0 . 1} \mathbf{~ m} / \mathbf{s}$ & $\mathbf{V}=\mathbf{1 . 2} \mathbf{~ m} / \mathbf{s}$ & $\mathbf{V = 0 . 1} \mathbf{~ m} / \mathbf{s}$ & $\mathbf{V = 1 . 2} \mathbf{~ m} / \mathbf{s}$ \\
VBF & 38,02 & 38,60 & 28,82 & 24,52 \\
HH-VBF & 38,28 & 38,48 & 22,58 & 12,04 \\
SBR-DLP & 14,90 & 13,76 & 13,26 & 8,16 \\
FBR & 36,32 & 37,80 & 34,80 & 15,32 \\
\hline
\end{tabular}

Tabla 3 Promedios Throughput (bps)

Fuente: Elaboración propia

En la figura 6, se puede observar que, en las Redes Dispersas, al aumentar la velocidad de los nodos, el Throughput disminuye con los protocolos VBF, HH-VBF y FBR mientras que con SBR-DLP tiende a aumentar ligeramente. 


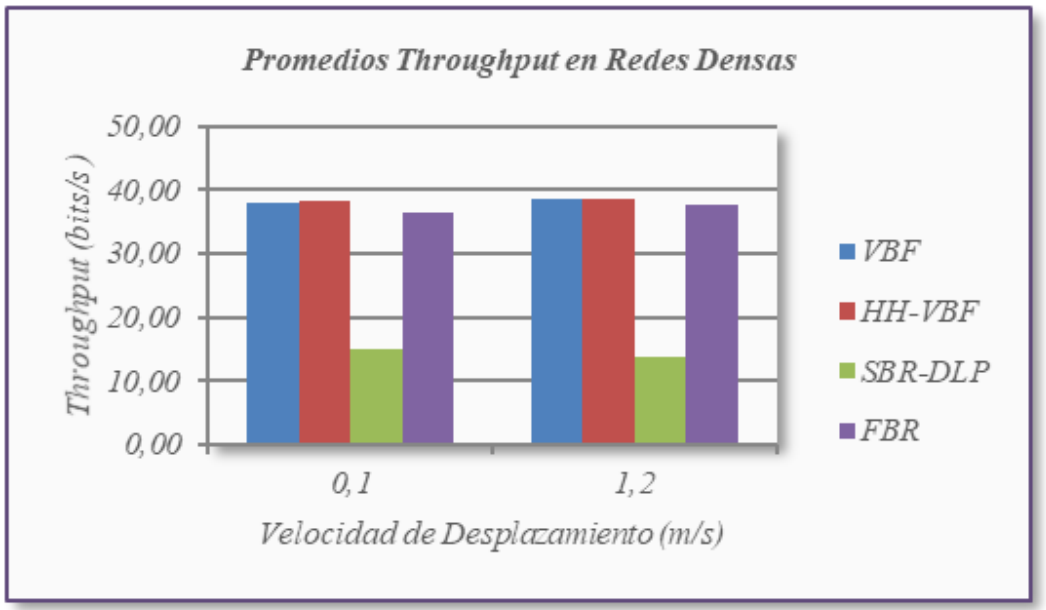

Figura 6 : Promedios Throughput dentro de las Redes Densas

Fuente: Elaboración propia

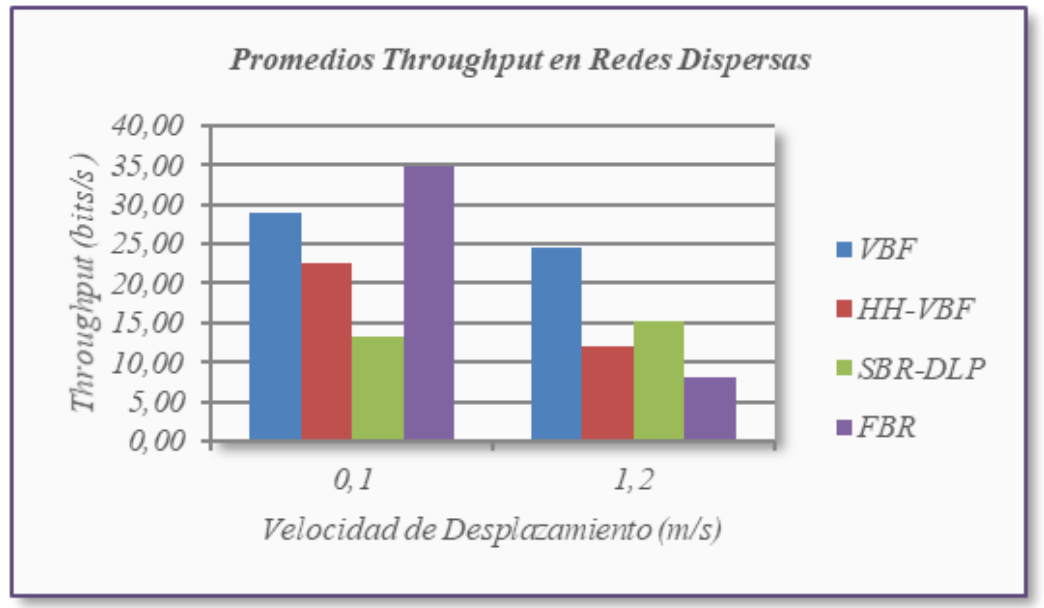

Figura 7 Promedios Throughput dentro de las Redes Dispersas

Fuente: Elaboración propia

Se realiza una prueba de normalidad Shapiro-Wilk de los datos obtenidos de la simulación, se ha utilizado la prueba de varianza Anova de un solo factor que permite determinar la existencia de diferencias entre las muestras obtenidas y para detectar qué muestras difieren se ha utilizado la prueba estadística complementaria post hoc de HSD de Tukey, se obtiene los resultados de tabla 4 en la que, dentro de cada parámetro de rendimiento analizado se ubica al protocolo que presenta los mejores resultados en cada uno de los escenarios planteados, con lo que se puede comprobar que la evaluación de los mecanismos de localización de nodos para redes UWSN a través de la herramienta de simulación Aqua-Sim ha permitido determinar el protocolo que combina valores óptimos de rendimiento y consumo de energía reducido, por lo que, la hipótesis planteada ha sido verificada y aceptada. 


\begin{tabular}{|c|c|c|c|c|}
\hline \multicolumn{2}{|c|}{$\begin{array}{c}\text { REDES DENSAS } \\
\text { MÓVILES }\end{array}$} & \multirow[t]{2}{*}{$\begin{array}{l}\text { PARÁMETRO DE } \\
\text { RENDIMIENTO }\end{array}$} & \multicolumn{2}{|c|}{$\begin{array}{c}\text { REDES DISPERSAS } \\
\text { MÓVILES }\end{array}$} \\
\hline $\mathrm{V}=\mathbf{0 , 1} \mathrm{m} / \mathrm{s}$ & $\mathrm{V}=1,2 \mathrm{~m} / \mathrm{s}$ & & $\mathrm{V}=\mathbf{0 , 1} \mathrm{m} / \mathrm{s}$ & $\mathrm{V}=1,2 \mathrm{~m} / \mathrm{s}$ \\
\hline VBF & VBF & End and End Delay & FBR & FBR \\
\hline VBF & VBF & Energy Consumption & FBR & FBR \\
\hline VBF & VBF & Throughput & FBR & FBR \\
\hline
\end{tabular}

Tabla 4 Resumen de la asignación de la posición de los protocolos Fuente: Elaboración propia

\section{Conclusiones.}

- Para arquitecturas de red UWSN densas, con nodos moviéndose a velocidades de $0.1 \mathrm{~m} / \mathrm{s}$ (época seca del año), el protocolo que presenta mejor rendimiento es VBF, ya que posee un retardo moderado (154,48 ms), throughput significativo (38.02 bits/s) mientras consume menos energía $(1,15402 \mathrm{~W} / \mathrm{s})$ que el resto de los protocolos analizados.

- Para arquitecturas de red UWSN densas con nodos moviéndose a velocidades de 1.2 $\mathrm{m} / \mathrm{s}$ (época húmeda del año), el protocolo que presenta mejor rendimiento es VBF con valores de retardo (149,67 ms), throughput (38.60 bits/s) y consumo de energía $(1,15701 \mathrm{~W} / \mathrm{s})$.

- Para arquitecturas de red UWSN dispersas, con nodos moviéndose a velocidades de $0.1 \mathrm{~m} / \mathrm{s}$ (época seca del año), el protocolo que presenta mejor rendimiento es FBR, con valores de retardo (4,60 ms), throughput (34.80 bits/s) y consumo de energía $(0,12148 \mathrm{~W} / \mathrm{s})$.

- Para arquitecturas de red UWSN dispersas con nodos moviéndose a velocidades de $1.2 \mathrm{~m} / \mathrm{s}$ (época húmeda del año), el protocolo que presenta mejor rendimiento es FBR con valores de retardo $(1,49 \mathrm{~ms})$, throughput $(15.32 \mathrm{bits} / \mathrm{s})$ y consumo de $(0,12156$ $\mathrm{W} / \mathrm{s})$.

- Al incrementar la densidad de los nodos mejora el promedio de entrega de paquetes, esto se da porque al existir más nodos existe más posibilidades de que éstos se encuentren dentro de la tubería virtual elegible, en otras palabras, más nodos son calificados para enviar paquetes.

- La transmisión inalámbrica de información a través del agua, el envío y recepción de mensajes por medio de un canal acústico, es decir, las UWSN se han convertido en 
una poderosa herramienta tecnológica para el desarrollo de importantes sistemas de observación oceanográfica a gran escala.

\section{Referencias bibliográficas.}

[1] Camp, T., Boleng, J., \& Davies, V. (2002). A survey of mobility models for ad hoc network research. Wireless communications and mobile computing, 2(5), 483-502.

[2] Chirdchoo, N., Soh, W. S., \& Chua, K. C. (2009, May). Sector-based routing with destination location prediction for underwater mobile networks. In 2009 International Conference on Advanced Information Networking and Applications Workshops (pp. 1148-1153). IEEE.

[3] Jornet, J. M., Stojanovic, M., \& Zorzi, M. (2008, September). Focused beam routing protocol for underwater acoustic networks. In Proceedings of the third ACM international workshop on Underwater Networks (pp. 75-82). ACM.

[4] Naranjo, C., \& Tapia, M. E. (2015). Plancton en el canal Bolívar de la isla Isabela (Caleta Tagus), Islas Galápagos durante marzo de 2009. Acta Oceanogr. del Pac, 20, 71-87.

[5] Nicolaou, N., See, A., Xie, P., Cui, J. H., \& Maggiorini, D. (2007). Improving the robustness of location-based routing for underwater sensor networks. Oceans 2007Europe, 18.

[6] Sistema Nacional de Áreas Protegidas del Ecuador. (2014). Reserva Marina Galápagos. Obtenido de http://areasprotegidas.ambiente.gob.ec/sites/default/files/GUIA_PARQUES_01-12014.pdf

[7] Xie, P., Zhou, Z., Peng, Z., Yan, H., Hu, T., Cui, J. H., ... \& Zhou, S. (2009, October). AquaSim: An NS-2 based simulator for underwater sensor networks. In OCEANS 2009 (pp. 1-7). IEEE. 


\section{PARA CITAR EL ARTÍCULO INDEXADO.}

Paucar Samaniego, J., Martínez Guashima, O., Navarrete Chávez, F., \& Cujilema Daga, G. (2019). Plataforma de Evaluación de Mecanismos de Localización de Nodos en Redes de Sensores Inalámbricos Bajo el Agua - UWSN. Ciencia Digital, 3(3.3), 162-176. https://doi.org/10.33262/cienciadigital.v3i3.3.777

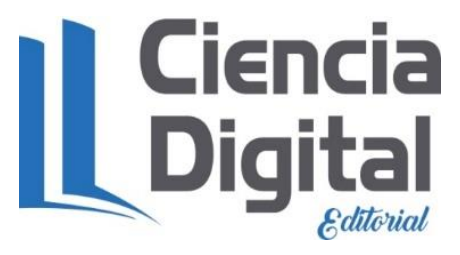

El artículo que se publica es de exclusiva responsabilidad de los autores y no necesariamente reflejan el pensamiento de la Revista Ciencia Digital.

El artículo queda en propiedad de la revista y, por tanto, su publicación parcial y/o total en otro medio tiene que ser autorizado por el director de la Revista Ciencia Digital.
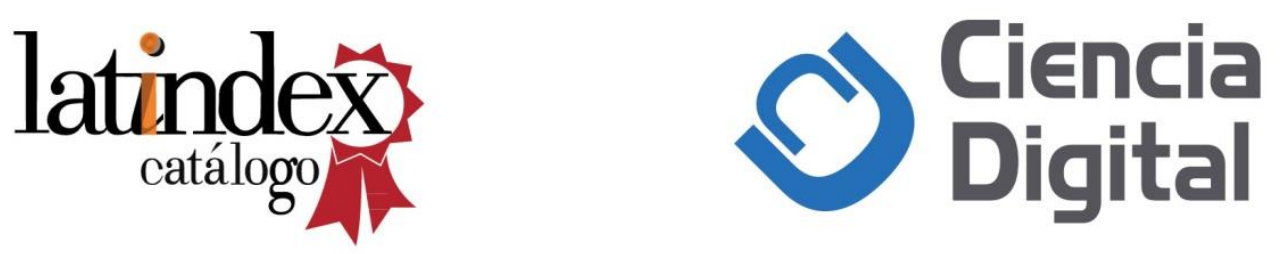